\title{
Using structural equation modelling to examine McCollough effects (orientation-contingent colour aftereffects): Influence of Dissociative experiences and age on illusory aftereffects
}

Peter Grimbeek, Griffith University, Graham Jamieson, University of New England and Kathryn Gow, Queensland University of Technology

\begin{abstract}
Conventional accounts of the McCollough Effect (ME) have focussed on strictly bottom-up processing accounts of the phenomenon, most commonly involving the fatiguing of orientation-selective neurons, although association-learning mechanisms have also gained acceptance. These lower-order accounts do not take into higher-order variables related to key personality traits and/or associated cognitive control processes. This article reports the use of confirmatory factor analysis (CFA) and follow-up structural equation style regressions that model MEs and also the part played by the personality trait of dissociation. After considering the relative impact of age and dissociative processes, the article concludes that trait dissociation is positively associated with reports of MEs.
\end{abstract}

\section{Introduction}

In 1965, Celeste McCollough reported that participants in her experiments observed illusory colour aftereffects contingent on orientation after viewing chromatic gratings. For instance, after viewing vertical edges paired with green, participants reported illusory red aftereffects on vertical edges.

This complementary colour effect, dubbed the McCollough Effect (ME) or less personably later on as the orientation-contingent colour aftereffect (OCCAE) was explained by her as a low-level phenomenon resulting from the neural fatigue of over-stimulated edge detectors in 
MEs \& dissociation: CFA \& SEM analyses

the visual system. Subsequent studies (e.g., Harris, 1980; Lovegrove \& Over, 1972; Riggs, Jones \& Holding, 1975; Skowbo, Gentry, Timney \& Morant, 1974; Stromeyer, 1969; White \& Eimas, 1974) have either embraced variants on this explanation (e.g., Stromeyer, 1972; Michael, 1978; cited in Houck \& Hoffman, 1986; Humphrey, 1998), developed association learning explanations (e.g., Murch, 1976; Allan \& Siegel, 1986) that implicitly rely on lowlevel cognitive mechanisms, or constructed explanations that blend these low-level explanations (Dodwell \& Humphrey, 1990; Bedford, 1995; Shaw, 2000).

To date, no higher-order modulation of the ME has been demonstrated convincingly. Finke and Schmidt $(1977,1978)$ reported that MEs could be induced subsequent to imagining oriented edges superimposed on fields of homogeneous colour. However, Broerse and Crassini (1980), after replicating this study, reported that not only did the act of imagining oriented edges not lead to the induction of MEs, but also severely questioned Finke and Schmidt's (1977) practice of excluding participants who reported association type strategies as a way to increase the quality of the outcomes. This practice, in effect, would have excluded many of the non-complementary aftereffects.

Broerse and Crassini (1980) also examined the effect of imagery ability on the MEs induced under standard induction conditions. They reported that the strength and direction of MEs were not predicted significantly by level of imagery ability. This outcome militated against higher- in favour of lower-order driving mechanisms, as has other work reported by these researchers (Broerse \& Crassini 1980; Also see Barber, 1959).

Erickson and Erickson (1938) examined another aspect of higher-order cognitive control, response to hypnotic suggestion, in relation to the induction of simple colour aftereffects (CAEs; See also Anstis, 1967; Blakemore \& Sutton, 1969; Gibson, 1937). Hypnotised participants hallucinated suggested colours on achromatic sheets of paper, and then reported illusory AE colours on subsequent achromatic sheets of paper. Four of the five participants reported appropriately complementary CAEs (the fifth was unable to hallucinate colours). For instance, after hallucinating a green hue, these participants reported the colour of the next 


\section{MEs \& dissociation: CFA \& SEM analyses}

sheet to be red. The intersection of Broerse and Crassini's (1980) negative outcomes with MEs and Erickson and Erickson's (1938) positive outcomes with CAEs suggests the appropriateness of blending the best and avoiding the weak points in each approach.

Some of the strengths in Erickson and Erickson's approach include the emphasis on the experience of hypnotic color hallucination as opposed to simple imagery ability and also the use of multiple induction colours. An advantage in adopting Erickson and Erickson's procedure of varying the induction colours is that if participants responses are being influenced by guessing the illusory aftereffect, then unannounced change in induction conditions are more likely to result in incorrect answers. For that reason, the current study has partially replicated Erickson and Erickson's approach in adopting a procedure of inducing and testing MEs four times under standard induction conditions, and then inducing and testing MEs under a complementary set of standard induction conditions a further four times.

However, a criticism of Erickson and Erickson's methodology is that it is easier to guess the association between induction and aftereffect colours under simple CAE conditions, as this knowledge is relatively commonplace. The ME is more difficult to guess, given the more tenuous link between orientation contingency at induction and at test. The tenuous nature of this link is in fact the weakest point in association learning explanations of MEs. That is, one would not necessarily expect standard MEs to emerge from the associative pairings of colours with orientations. For that reason, the present study has tested the effect of hypnotic susceptibility with illusory colours induced under standard ME conditions, rather than under the simple CAE conditions favoured by Erickson and Erickson.

Comparatively, the strengths in Broerse and Crassini’s (1980) study included their interest in not only examining the effect of imagery ability on MEs under imaginary orientation conditions, but also under standard induction conditions. While the reliance on imaginary induction conditions paralleled those employed both by Finke and Schmidt $(1977,1978)$ and earlier by Erickson and Erickson (1938), it seems more likely that higher-order modulation effects would be observed under actual induction conditions than under pseudo induction 


\section{MEs \& dissociation: CFA \& SEM analyses}

conditions. Evidence in favour of this proposition includes not only studies reported by Broerse and Crassini but also Thompson and Latchford's (1986) findings that CAEs are contingent on the wavelength of veridical sensory stimuli. For that reason, the current study has examined the effect of trait dissociation, itself linked to hypnotic susceptibility, under standard ME induction conditions rather than the pseudo induction conditions favoured elsewhere.

In summary, this study has hypothesised that susceptibility to dissociative states (characteristic of high susceptibles in hypnosis), as measured by the Dissociative Experiences Scale Two (DESII) will predict the strength and direction of MEs under conditions in which one standard induction (repeated four times) is followed immediately by a complementary standard induction (repeated four times).

\section{Methods}

This study used data collected over a two-year period by a group of students engaged in postgraduate research activities. A companion article reports the results of analysing outcomes from the two years separately (Jamieson, Grimbeek, Carol-Walden \& Gow, submitted). The 129 participants ranged in age from 17 to 55 years, with a mean age of 25 years. Of the 129 participants, $84 \%$ were female, with the distribution of ages positively skewed towards the early 20s.

The procedure employed by these students was to induce MEs by briefly exposing groups of first year psychology students to PowerPoint slides containing horizontal and red edges, paired with red and green hues respectively, as per displays utilised previously by Broerse and Crassini (1980). The students gave two successive reports of illusory colours on achromatic vertical and horizontal edges, on reversed versions of a test display based on that used by Broerse and Crassini (1980). This induction-test procedure was repeated four times, after which the pairings of colours with orientations was reversed (say, red verticals instead of green verticals), and the same induction-test procedure repeated for another four times. For a 
detailed version of the methods employed see the companion article (Grimbeek, Jamieson, Carol-Walden \& Gow, submitted).

These participants were subsequently required to complete a questionnaire that collected not only demographic details (gender, age, etc.), but also responses to items in the DESII (Dissociation experiences scale).

\section{Results}

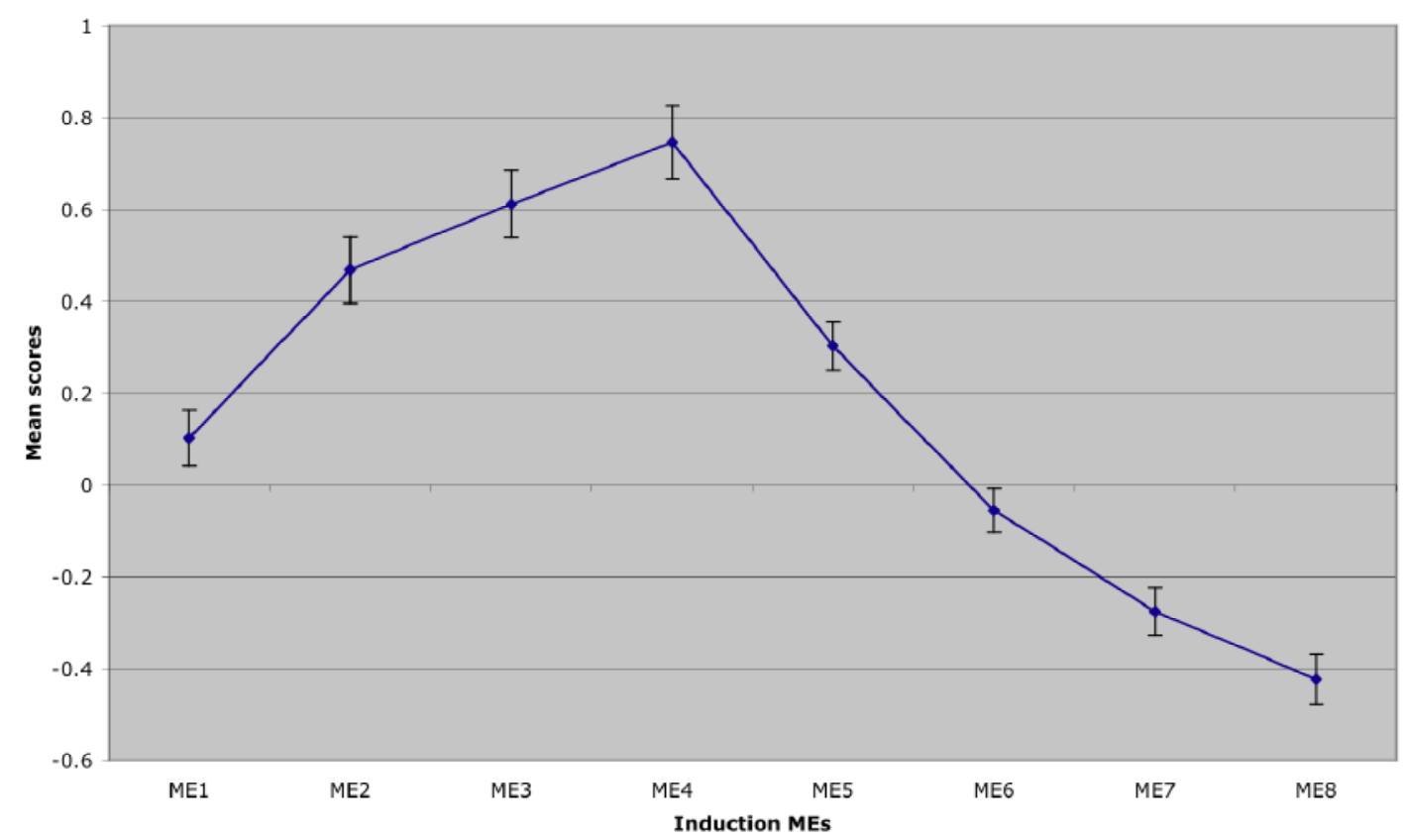

Figure 1 Average strength and direction of MEs reported across eight induction-test sessions

Participant reports of MEs were collated and coded such that colours, complementary to those observed during the initial set of inductions, were scored positively (i.e., after inspecting red verticals, reports of observing green with a magnitude of two were scored as +2 ) for the entire eight sessions. Given a lack of conceptual interest in whether these illusory colours were observed at the centre or periphery, or in the first of two displays versus the second, participant responses were averaged across the four CAE reports obtained at each test session. As shown in Figure 1, the strength of MEs increased across the first four sessions in a standard fashion and then declined across the next four sessions consistent with the reversed 
MEs \& dissociation: CFA \& SEM analyses

pairings of colour to orientation (the effect of reversing contingencies being to cancel the initial effects and then induce effects in the opposite direction).

Participant scores on the DESII varied ranged from 1.43 to 56.07 , with a median score of 13.21. That is, scores were positively skewed such that most participants obtained lower, rather than higher, scores.

\section{Confirmatory factor analysis for the two phases of ME induction}

Confirmatory factor analysis (CFA) was used to examine the extent to which MEs induced in sessions 1-4 and 5-8 expressed unidimensional latent variable consistent with the MEs predicted from the two phases of induction (where Model A CFA is based on Sessions 1-4, Model B CFA is based on Sessions 5-8). After excluding eight participants based on Mahalanobis distance values, Mardia's coefficient of multivariate kurtosis was reduced to 22.841 for the Model A CFA and to 12.642 for the Model B CFA, respectively.

Table 1 Estimates of Goodness of Fit for two confirmatory factor analyses and a regression-style structural equation follow-up analysis (no structural elements)*

\begin{tabular}{|c|c|c|c|c|}
\hline Model & $\begin{array}{c}\text { Model A } \\
\text { CFA }\end{array}$ & Model B CFA & $\begin{array}{c}\text { Effect of } \\
\text { dissociation \& } \\
\text { age on Model A } \\
\text { and B MEs }\end{array}$ & Ideal \\
\hline Chi square & .769 & 1.294 & 1.590 & \\
\hline Degrees of freedom (df) & 1 & 1 & 2 & \\
\hline Probability & .380 & .255 & .452 & $\mathrm{p}>0.05$ \\
\hline Chisquare/df & .769 & 1.294 & .795 & $0.00-3.00$ \\
\hline $\begin{array}{l}\text { Root mean square residual } \\
\text { (RMR) }\end{array}$ & .005 & .005 & .276 & $0.00-0.05$ \\
\hline Normed fit index (NFI) & .998 & .996 & .985 & $0.95-1.00$ \\
\hline Relative fit index (RFI) & .986 & .978 & .956 & $0.95-1.00$ \\
\hline
\end{tabular}


MEs \& dissociation: CFA \& SEM analyses

\begin{tabular}{lcccc}
\hline Tucker-Lewis index (TLI) & 1.005 & .995 & 1.012 & $0.95-1.00$ \\
Comparative fit index & 1.000 & .999 & 1.000 & $0.95-1.00$ \\
$(\mathrm{CFI})$ & & & & \\
Root mean square error of & .000 & .049 & .000 & $0.00-0.06$ \\
approximation (RMSEA) & & & & \\
\hline
\end{tabular}

*Less than ideal values bolded

In both models, after inspection of the modification index, an explicit link was included between the first two test sessions (1-2, 5-6) to take account of the redundancy of error terms associated with these two estimates. As indicated in Table 1, under these conditions, the entire range of estimates of goodness of fit for these two models was highly acceptable (for further details about measures see Byrne, 2001).

AMOS provides a range of goodness of fit estimates, some of which are mentioned here. The Chi-Square value is the most highly regarded of these, with one view being that the significance or non-significance of the Chi Square statistic is the only worthwhile measure of goodness of fit (Barrett, 2007). An issue with this approach is that the Chi Square is well known to be sensitive to sample size. That is, the larger the sample, the more likely this statistic is to be significant. An ancillary measure that attempts to correct for this is obtained by dividing the Chi by the number of degrees of freedom. This produces a Chi-Square statistic corrected for model complexity, with acceptable scores in the range of 0.00 to 0.30 . Second, the Root Mean square Residual (RMR) compares the hypothesised to the obtained covariance matrix, with the acceptable fit being equal to or less than 0.05 . Third, the Normed Fit Index (NFI), Tucker-Lewis index (TLI), and Comparative Fit Index (CFI) compare the default with the independence (baseline) model, with the acceptable level for each of these being equal to or greater than 0.90-0.95 (see Byrne, 2001). Finally, the Root Mean Square Error of Approximation (RMSEA) compares the hypothesised to the saturated model, with an acceptable fit being equal to or less than 0.06.An initial step is to examine the effect of dividing the chi square value (CMIN) by the degrees of freedom (DF). This operation results 
MEs \& dissociation: CFA \& SEM analyses

in a ratio (CMIN/DF) with an ideal value of 0 and a threshold of acceptance at the level of 3.

Both models exhibited a value in the acceptable 0-3 range.

Table 2 Standardised regression weights for Models A and B (All at $p<0.001$ level)

\begin{tabular}{|l|c|c|c|c|c|c|c|c|}
\hline IV & \multicolumn{4}{|c|}{ MODEL A } & \multicolumn{4}{c|}{ MODEL B } \\
\hline DV & Session & Session & Session & Session & Session & Session & Session & Session \\
& 1 & 2 & 3 & 4 & 5 & 6 & 7 & 8 \\
\hline Beta & 0.651 & 0.872 & 0.900 & 0.919 & 0.473 & 0.808 & 0.995 & 0.866 \\
weight & & & & & & & & \\
\hline
\end{tabular}

The examination of estimates of fit was supplemented by an examination of the size of standardised regression weights (all MEs significantly predicted by the two ME models). It is clear from the above that these standardised regression weights were all in the moderate to strong range of values.

A supplementary examination of standardised residuals indicated that most of the residuals approximated zero and none exceeded 1 as a value. In short, none of the standardised residuals exhibited extreme values.

\section{Modelling the effect of demographic variables on MEs}

An initial consideration was the presence of a significant negative correlation between age in years and DESII score (Pearson's Correlation Coefficient $(N=120)=-0.246, \mathrm{p}<0.01)$ (that is, younger participants tended to obtain higher dissociation scores and vice versa). For this reason, the SEM explicitly modelled the associations between dissociation, age, and reports of MEs.

As the distribution on the DESII was positively skewed, as noted above, this variable was transformed (natural log) to reduce the level of skew, and its association with the eight MEs examined via structural equation modelling (SEM). Five participants did not report their age, and were allocated the average age instead (in order to maximise the number of cases entered into the analysis). 
Given that sessions 1 and 5 were only moderately associated with their respective latent variables (See Figure 1), these two sessions were discarded in subsequent analyses.
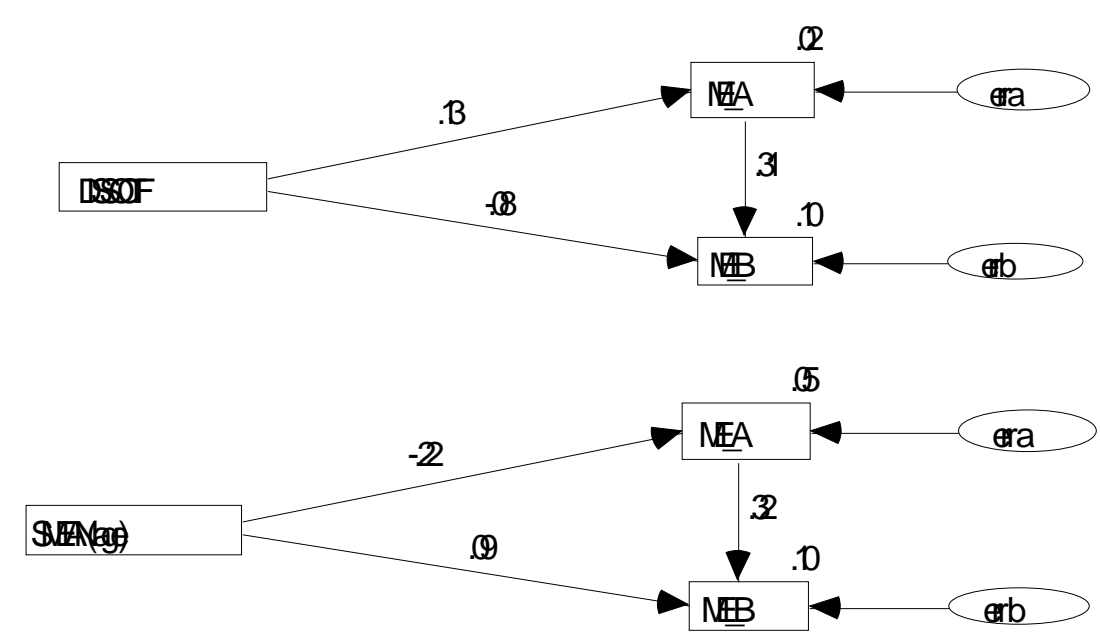

Figure 2 Follow-up saturated models illustrating strength of associations between dissociation, age, and MEs induced in sessions 2-4 (ME_A) versus 6-8 (ME_B)

The initial plan was to utilise the two ME models tested by CFAs to model the association between dissociation level and age in years. These models, illustrated in Figure 2, resulted in saturated models and nonsignificant associations, other than for age in relation to Model A (Sessions 2-4). Given, the paucity of significant effects for Model B, a follow-up analysis of the combined effect of dissociation and age (Figure 3) focused on a more detailed scrutiny of sessions 1-4.

After eliminating nonsignificant linkages and variables (i.e., ME3, ME4), age turned out to be significantly (negatively) associated with ME2, whereas dissociation was significantly (positively) associated with ME1 (albeit with low coefficients), as illustrated in Figure 3.

Examination of the values shown in Table 1 indicates a range of acceptable fit values with the exception of RMR, which indicated that the model illustrated in Figure 3 did not adequately explain all of the variance. However, RMSEA indicated to the contrary that this model was highly effective in terms of its use of the available variance. 
MEs \& dissociation: CFA \& SEM analyses

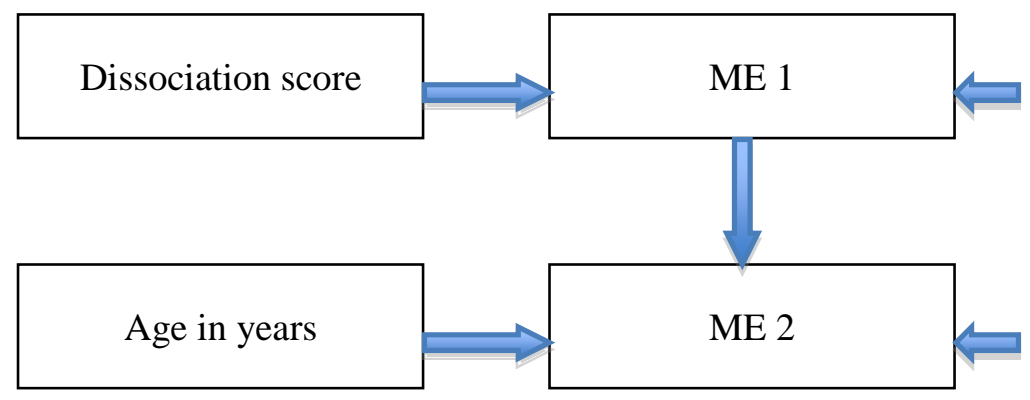

Figure 3 Regression-style model for dissociation and age in relation to MES induced in sessions 1 (ME 1) and 2 (ME 1)

Table 3 Standardised regression weights for Dissociation and age in relation to MES induced in sessions 1 (ME1) and 2 (ME2)

\begin{tabular}{|lcc|}
\hline IV & DV & Beta weight \\
\hline Dissociation score & ME1 & $0.188^{*}$ \\
ME1 & ME2 & $0.680^{* * *}$ \\
Age in years & ME2 & $-0.139^{*}$ \\
\hline Note: ${ }^{* * *}=p<0.001,{ }^{* *}=\mathrm{p}<0.01,{ }^{*}=\mathrm{p}<0.05$ & & \\
\hline
\end{tabular}

Table 3 indicates these beta weights, with the exception of the link between successive MEs to be weak but nonetheless statistically significant.

\section{Discussion}

This article set out to examine the extent to which the separate sets of MEs induced by experimental sessions 1-4 and sessions 5-8 could be modelled via confirmatory factor analyses. It also set out to examine the significance of the association between trait dissociation and these MEs.

As shown in Table 2, MEs could be modelled successfully via SEM's confirmatory factor analytic procedures, with the range of statistical values indicating the quality of the model in question, though undoubtedly with a lesser degree of reliability given that the sample size did not exceed 100. These analyses are however conceptually important, in that they point the way to examining the underlying categories governing the response of participants to illusory 


\section{MEs \& dissociation: CFA \& SEM analyses}

colours on achromatic edges, as opposed to making the arbitrary assumption that responses do, in fact, cluster in a unidimensional manner. Further, this statistical approach to analysing participant reports of MEs, in principle, has the virtue of facilitating follow-up analyses that use these identified factors to model the associations between MEs and other phenomena,

As illustrated in Figure 2, and highlighted via Tables 2 and 3, the analytic procedures utilised in this paper allow one to assess the complexity of the influences bearing on participant selfreports. While both the age range and the clinical normalcy of the sample represent natural limitations to the power of the present set of analyses, they nonetheless hint at the contrary effects of increasing age and increasing levels of dissociation in relation to successive reports of illusory colour aftereffects.

The differing nature of the significant associations between Dissociation and Age in relation to Sessions 1 vs. 2, as illustrated in Figure 3, were mirrored (See Figure 2) in the largely nonsignificant SEMs for Model A (sessions 2-4) vs. Model B (session 6-8). What these saturated models suggest is that the trends reported for Session 1 and 2 generalise across the eight induction-test sessions. That is, regardless of induction conditions one might expect older participants to be less likely to report the predicted MEs (i.e., negative beta weight in sessions 2-4; positive in sessions 6-8), whereas participants with higher levels of dissociation would be more likely to report the predicted ME (i.e., positive beta weights in sessions 2-4 and negative in sessions 6-8).

In that regard, it is of interest that the instrument for measuring dissociation here relied on is negatively correlated with age. This significant negative correlation provides an alternative explanation for this measure of dissociation (DESII), that is, lower levels of dissociation here appear to reflect something in the aging process rather than an orthogonal cognitive factor. This methodological problem indicates the need in future research to utilise measures of higher order control (perhaps hypnotic susceptibility itself), which are highly stable with age in the adult population (Piccione, Hilgard \& Zimbardo, 1989). 


\section{MEs \& dissociation: CFA \& SEM analyses}

These tentatively positive conclusions also support the decision in the present study to examine the effect of higher-order concomitants of MEs under standard, rather than imaginary, induction conditions. The notion of examining the ability to imagine some kind of illusory aftereffect, after observing nothing but edges or nothing but colours appears problematic, in that it seems far more reasonable to assume that the activation of higher-order neurophysiological (cognitive) mechanisms requires bottom-up sensory input to function than to expect "something from nothing". In retrospect, it is not surprising that the outcomes of ME studies utilising nonveridical induction conditions reported either weak (Finke \& Schmidt, 1977, 1978) or negative outcomes (Broerse \& Crassini, 1980).

Probably the greatest procedural obstacle to obtaining high quality results in the present study was that the response sheet used for collecting participant reports of MEs included the option of responding as unsure or undecided, instead of reporting either green or red with some degree of certainty. In consequence, around $50 \%$ of participants from data collected in the second year of this study selected the undecided option, effectively distorting the distribution of data, and moving the mean towards the zero point, especially in sessions 5-8.

In conclusion, given the elusive but consistent trends reported here, it seems very likely that partial replications of the above with at least 200-300 participants to increase the effect sizes, and with the undecided option removed from response sheets, so that participants must make a choice of illusory colour aftereffects even when uncertain, would lead to greater support for the tentative conclusion at present that individual differences in personality traits such as dissociation (or associated variability in top-down cognitive control processes) do play a part in the ME.

\section{References}

Allan, L. G. \& Siegel, S. (1986). McCollough effects as conditioned responses: Reply to Skowbo. Psychological Bulletin, 100, 388-393.

Anstis, S. M. (1967). Visual adaptation to gradual change of intensity. Science, 155, 710-712. 


\section{MEs \& dissociation: CFA \& SEM analyses}

Barber, Theodore Xenophon (1964). Hypnotically Hallucinated Colors and Their Negative After-Images. The American Journal of Psychology, 77 ( 2), 313-318.

Barber, J. (1991). The locksmith model: Accessing hypnotic responsiveness. In S. J. Lynn \& J. W. Rhue (Eds.), Theories of hypnosis: Current models and perspectives (pp. 241-274). New York: Guilford Press.

Bedford, F. L. (1995). Constraints on perceptual learning: Objects and dimensions. Cognition, 54, 253-297.

Blakemore, C. \& Sutton, P. (1969). Size adaptation: A new aftereffect. Science, 166, 245247.

Broerse, J. \& Crassini, B. (1980). The influence of imagery ability on colour aftereffects produced by physically present and imagined stimuli. Perception and Psychophysics, 28, $560-568$

Byrne, B. M. (2001). Structural equation modeling with AMOS: Basic concepts, applications, and programming. Mahwah, NJ: Lawrence Erlbaum and Associates.

Carlson, E. B. \& Putnam, F. W. (1993). An update on the dissociative experiences scale. Dissociation, 6, 16-27.

Dodwell, P. C. \& Humphrey, G. K. (1990). A functional theory of the McCollough effect. Psychological Review, 97, 78-89.

Dodwell, P. C. \& Humphrey, G. K. (1993). What is important about McCollough effects? Reply to Allan and Siegal. Psychological Review, 100, 347-350.

Erickson, M. H. \& Erickson, E. M. (1938). The hypnotic induction of hallucinatory colour vision followed by pseudo negative after-images. Journal of Experimental Psychology, 22, 580-588.

Finke, R. A. \& Schmidt, M. J. (1977). Orientation-specific color aftereffects following imagination. Journal of Experimental Psychology: Human Perception and Performance, 3, 599-606. 


\section{MEs \& dissociation: CFA \& SEM analyses}

Finke, R. A. \& Schmidt, M. J. (1978). The quantitative measure of pattern representation in images using orientation-specific color aftereffects. Perception and Psychophysics, 23, 515-520.

Gibson, J. J. (1937). Adaptation with negative after-effect. Psychological Review, 44, 222244.

Harris, C. S. (1980). Visual coding and adaptability. NJ: Lawrence Erlbaum.

Houck, M. R. \& Hoffman, J. E. (1986). Conjunction of color and form without attention: Evidence from an orientation-contingent color aftereffect. Journal of Experimental Psychology: Human Perception and Performance, 12, 186-199.

Humphrey, G. K. (1998). The McCollough effect. In V. Walsh \& J. Kulikowski (Eds.), Why things look as they do? (pp. 32-61). Melbourne: Cambridge University Press.

Jamieson, G. A., Grimbeek, P., Carol-Walden, M. \& Gow, K. (submitted Personality and Individual Differences). Personality traits and the McCollough Effect: Dissociative experiences influence these orientation contingent colour aftereffects

Jones, P. D. \& Holding, D. H. (1975). Extremely long-term persistence of the McCollough effect. Journal of Experimental Psychology: Human Perception and Performance, 1, 323-327.

Lovegrove, W. J. \& Over, R. (1972). Color adaptation of spatial frequency detectors in the human visual system. Science, 176, 541-543.

McCollough, C. (1965). Color adaptation of edge-detectors in the human visual system. Science, 149, 1115-1116.

Murch, G. M. (1976). Classical conditioning of the McCollough effect: Temporal parameters. Vision Research, 16, 615-619.

Piccione, C., Hilgard, E. R., \& Zimbardo, P. G. (1989). On the degree of stability of measure hypnotizability over a 25-year period. Journal of Personality \& Social Psychology, 56 (2), 289-295. 
MEs \& dissociation: CFA \& SEM analyses

Riggs, L. A., White, K. D. \& Eimas, P. D. (1974). Establishment and decay of orientationcontingent aftereffects of color. Perception and Psychophysics, 16, 535-542.

Shaw, C. (2000). Are contingencies between edges and colour in perceptual aftereffects mediated by learned associations between orientation and colour? PhD thesis: School of Psychology, University of Queensland, Australia.

Skowbo, D., Gentry, T., Timney, B. \& Morant, R. B. (1974). The McCollough effect: Influence of several kinds of visual stimulation on decay rate. Perception and Psychophysics, 16, 47-49.

Stromeyer, C. F. III (1969). Further studies of the McCollough effect. Perception and Psychophysics, 6, 105-110.

Stromeyer, C. F. III (1972). Edge-contingent color after effects: Spatial frequency specificity. Vision Research, 12, 717-733.

Thompson P. \& Latchford, G. (1986). Colour-contingent after-effects are really wavelengthcontingent. Nature, 320, 525-526. 